\title{
Postop monitoring: on the record?
}

During the semiannual IACUC facility inspection at Great Eastern University, the inspection team visited the laboratory of Greg Morrison, DVM. As part of his IACUC-approved research, Morrison had implanted skull electrodes in rats. The procedure was relatively standard. Under general anesthesia and using aseptic technique, he made a small skin incision and then used a fine drill and bit to make holes in the cranium near the bregma. Then he inserted the electrodes and secured them with bone cement. The protocol stated that analgesia would be given if needed. The inspection team found the animals to be in good health and simply asked to see the operative and postoperative records. Morrison's lab technician produced the anesthesia records but said that there were no records of postoperative observations or analgesics used because all of the animals did fine postoperatively and he didn't have to use any analgesics. According to Morrison's instructions to him, the tech- nician said that no medical record entries were required unless an animal had a specific problem or required analgesia.

The site visit team didn't know what to do, so it deferred to the full Committee. The Attending Veterinarian (AV) felt strongly that monitoring data should be part of an animal's postoperative care record. He said that not only was it common sense, but such records were required under the Animal Welfare Act Regulations (AWRs), USDA Policy \#3, the Guide for the Care and Use of Laboratory Animals (Guide), and a 25 October 2004 'white paper' from the American College of Laboratory Animal Medicine (ACLAM) entitled Medical Records for Animals Used in Research, Teaching and Testing. The AV finished by saying that it would be impossible for the IACUC to determine whether proper postoperative care was provided if there were no records for review. There were some IACUC members who disagreed with the AV, saying that if they had to maintain health or postoperative monitoring records for every mouse or rat in their colony, they could never complete their work. After the discussion, the Committee voted to support the AV and require Morrison to maintain postoperative monitoring and care records. When the decision was presented to Morrison, he was incensed. He could not believe that the IACUC would not trust a veterinarian to provide proper postoperative care; more importantly, however, he countered that the AWRs do not apply to his research because he used rats. Furthermore, the other documents cited by the AV and IACUC were guidelines, not requirements. He had no objection to the IACUC or the AV observing any of his procedures or examining his animals, but he steadfastly refused to record normal findings, saying that it was unnecessary, not legally required, and a waste of his time.

Does the AV or the IACUC have the authority to compel Morrison to keep the medical records they believe to be appropriate? What can the IACUC do to prevent such problems in the future?

\section{RESPONSE}

\section{Compromise is in order}

\section{David M. Kurtz, DVM, PhD, DACLAM}

This is a common problem. What to do when the regulations are not explicitly stated, and the investigator says, "If it is not in the regulations, I don't have to do it"? Morrison is correct in stating that his work is not covered by the AWRs. However, if his work is funded by the Public Health Service (PHS), his studies are subject to the Health Research Extension Act of 1985. This law requires that "animal care committees ... assure compliance with the guidelines established...” including “... appropriate pre-surgical and post-surgical veterinary medical and nursing care...." The guidelines referred to in this Act are those set forth in the PHS Policy on Humane Care and Use of Laboratory Animals (PHS Policy) and the Guide. If the PHS does not fund his work but his institution has filed assurance with the Office of Laboratory Animal Welfare (OLAW), and/or the Association for Assessment and Accreditation of Laboratory Animal Care International (AAALAC International) accredits his institution, then the IACUC really does have the final say in this matter (not just the $\mathrm{AV})$. The IACUC does have the authority to compel Morrison to keep medical records they believe to be appropriate.

The main point is that the IACUC must assure compliance. What is the best mechanism to assure compliance that can withstand external review? If outside review- ers can 'easily' follow the mechanisms for IACUC oversight and find that oversight to be appropriate, the IACUC is doing its job. Everyone close to the field of laboratory animal science would agree that a veterinarian can be trusted to provide appropriate postoperative medical care (blatant bias intended). However, the most appropriate manner to 'assure' that appropriate postoperative care is given would be to maintain records. The AV is correct in stating that "... it would be impossible for the IACUC to determine whether proper postoperative care was provided if there were no records for review." Maintaining medical records for rodents is new, but times are changing. We can no longer use the excuse that the volume of paperwork would impede research and thus these species do not require the same level of oversight. 